\title{
ON THE SIZE OF THE BRAUER GROUP
}

ARMAND BRUMER AND MICHAEL ROSEN ${ }^{1}$

It is well known that the isomorphism classes of finite-dimensional central division algebras over a field $K$ form a group $B(K)$, called the Brauer group of $K$. This group may be described cohomologically as follows: let $G_{K}$ be the Galois group of the separable closure $K_{\mathrm{s}}$ of $K$, then $B(K)=H^{2}\left(G_{K}, K_{s}^{*}\right)$ where the cohomology is that of profinite groups [3]. This group is an important arithmetic invariant which has been studied in various contexts.

An examination of known cases has led us to the conjecture that if $B(K)$ has finite exponent, then either the exponent is two or $B(K)=(0)$. A somewhat stronger statement seems to be true. We write $A_{p}$ for the $p$-primary component of any abelian group $A$.

Conjecture. Either $B(K)_{p}$ contains a nontrivial divisible subgroup or $2 B(K)_{p}=(0)$. In particular, if $p$ is odd, the second alternative implies $B(K)_{p}=(0)$.

In another publication [1] Auslander and Brumer have shown the conjecture is true for function fields in $n \geqq 1$ variables over arbitrary ground fields. The purpose of this note is to provide some additional evidence for the conjecture based on the properties of pro- $p$ groups.

Lemma 1. If $p$ is the characteristic of $k$, then $B(K)$ is divisible by $p$.

Proof. In [3, Chapter II] it is shown that $\operatorname{cd}_{p} G_{K} \leqq 1$. Consider the short exact sequence

$$
(1) \rightarrow K_{s}^{*} \stackrel{p}{\rightarrow} K_{s}^{*} \rightarrow K_{s}^{*} / K_{s}^{* p} \rightarrow(1) .
$$

We must have $H^{2}\left(G_{K}, K_{s}^{*} / K_{s}^{* p}\right)=(0)$. Thus the map

$$
B(K) \stackrel{p}{\rightarrow} B(K)
$$

is onto.

This lemma shows that for our problem we may assume $p$ is different from the characteristic of $K$. We do so from now on.

Let $\mu$ be the group of all $p^{n}$ th roots on unity $n=1,2,3, \cdots$ contained in $K_{s}$.

Received by the editors March 19, 1967.

1 The work of the second author has been partially supported by NSF Grant GP-5435. 
Lemma 2. (a) $B(K)_{p} \approx H^{2}\left(G_{K}, \mu\right)$. (b) For each subgroup $H$ of $G_{K}$, $H^{1}(H, \mu)$ is divisible.

Proof. The group $K_{s}^{*} / \mu$ is uniquely $p$ divisible, hence the $p$ primary part of its cohomology vanishes in dimensions greater than 0 . The short exact sequence (1) $\rightarrow \mu \rightarrow K_{s}^{*} \rightarrow K_{s}^{*} / \mu \rightarrow(1)$ thus implies the truth of the first assertion.

Let $\mu_{p}$ be the group of $p$ th roots of unity and consider the commutative diagram.

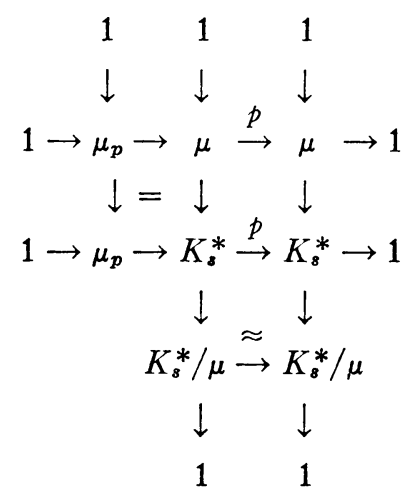

In cohomology we have

$$
\begin{aligned}
& H^{1}(H, \mu) \stackrel{p}{\rightarrow} H^{1}(H, \mu) \rightarrow H^{2}\left(H, \mu_{p}\right) \rightarrow H^{2}(H, \mu) \\
& \downarrow \quad \downarrow \quad \downarrow=\downarrow \approx \\
& H^{1}\left(H, K_{s}^{*}\right) \rightarrow H^{1}\left(H, K_{s}^{*}\right) \rightarrow H^{2}\left(H, \mu_{p}\right) \rightarrow H^{2}\left(H, K_{8}^{*}\right)_{p} .
\end{aligned}
$$

By Hilbert's Theorem 90, $H^{1}\left(H, K_{s}^{*}\right)=(0)$. The second assertion follows at once.

Lenma 3. Suppose $G$ is a pro-nilpotent group and $P$ its p-Sylow subgroup, then either $H^{2}(G, \mu) \approx H^{2}(P, \mu)$ or $H^{2}(G, \mu)=(0)$.

Proof. Since $G$ is pro-nilpotent, $P$ is a direct factor of $G, G=P N$. The order of $N$ is prime to $p$. Thus $H^{m}(N, \mu)=(0)$ for all $m>0$. From the Hochschild-Serre spectral sequence, we see $H^{2}\left(P, \mu^{N}\right) \approx H^{2}(G, \mu)$. The automorphism group of $\mu$ is isomorphic to $U$, the group of $p$-adic units. From this and the fact that the order of $N$ is prime to $p$, one sees that $\mu^{N}$ is either (1) or $\mu$. The conclusion follows.

Lemma 4. Suppose $G=G_{K}$ is an abelian pro-p-group and that $\sqrt{ }(-1) \in K$ if $p=2$. Then one of the following must hold.

(i) $G$ acts trivially on $\mu$.

(ii) $G$ is isomorphic to the p-adic integers, i.e. cd $G=1$. 
Proof. A torsion element of $G$ will define a subfield of finite codimension in the algebraic closure of $K$. Under our hypotheses the well-known theorem of Artin-Schreier shows there can be no such subfield. Thus $G$ is torsion free.

As has already been mentioned, the automorphism group of $\mu$ is isomorphic to the $p$-adic units. $G$ is a pro- $p$-group. Hence the image of $G$ in $\operatorname{Aut}(\mu)$ is either trivial or isomorphic to the $p$-adic integers. If neither (i) nor (ii) hold, we can find a subgroup $H=\langle\sigma, \tau\rangle$ of $G$ such that $\sigma$ acts trivially on $\mu$ and $\tau$ acts nontrivially. The Hochschild-Serre spectral sequence yields

$$
(0) \rightarrow H^{1}(\langle\tau\rangle, \mu) \rightarrow H^{1}(H, \mu) \rightarrow H^{1}(\langle\sigma\rangle, \mu)^{\langle\tau\rangle} \rightarrow H^{2}(\langle\tau\rangle, \mu) .
$$

The last term vanishes since $\operatorname{cd}\langle\tau\rangle=1$. Since $\sigma$ acts trivially on $\mu$, $H^{1}(\langle\sigma\rangle, \mu)=\operatorname{Hom}(\langle\sigma\rangle, \mu) \approx \mu$. Hence the next to last term is isomorphic to the group of elements of $\mu$ left fixed by $\tau$. This group contains at least $\mu_{p}$ but is finite since $\tau$ acts nontrivially.

By Lemma $2, H^{1}(H, \mu)$ is divisible. We have now arrived at a contradiction since the epimorphic image of a divisible group cannot be a nontrivial finite group.

Lemma 5. Let $F$ be a free pro-p-group and $R$ a closed normal subgroup. If $R \neq(1)$, then $R /[R, F]$ is not a torsion group.

Proof. Let $F_{0}=F$ and define $F_{k+1}=\left[F_{k}, F\right]$. It is known that $F_{k} / F_{k+1}$ is torsion free (cf. for instance Proposition 2 of [2]). Since $\cap F_{k}=(1)$ our hypotheses imply that for some $k$ we have $R \subset F_{k}$ but $R \nsubseteq F_{k+1}$. Since $[R, F] \subset F_{k+1}$, the natural map yields a nontrivial homomorphism

$$
R /[R, F] \rightarrow F_{k} / F_{k+1},
$$

$F_{k} / F_{k+1}$ is torsion free, so $R /[R, F]$ cannot be torsion.

Theorem. Let $K$ be a field containing all $p^{n}$ th roots of unity, $n$ $=1,2,3, \cdots$. Let $L$ be the maximal pextension of $K$, and let $P$ be the Galois group of $L$ over $K$. Then one of the following must hold:

(i) $B(K)_{p}$ contains a nontrivial divisible subgroup.

(ii) $P$ is a free pro-p-group, so that $B(L / K)=(0)$.

Proor. Consider the subgroup $B(L / K)=H^{2}\left(P, L^{*}\right)$ of $B(K)$ consisting of those elements split by $L$. Since $\mu \subset K^{*}$ and $L$ has no $p$ extensions, we can apply the considerations of Lemma 2 to conclude that $B(L / K) \approx H^{2}(P, \mu)$ and $H^{1}(P, \mu)$ is divisible. $P$ acts trivially on $\mu$, so $H^{1}(P, \mu) \approx\left(P / P_{1}\right)^{\wedge}$, the character group of $P / P_{1}$. Pontryagin duality implies $P / P_{1}$ is torsion free. 
Represent $P$ as the quotient $F / R$ of a free pro-p-group $F$ by a closed normal subgroup $R$. We may suppose $F$ is minimal (cf. [3]). The Hochschild-Serre sequence gives

$$
(0) \rightarrow H^{1}(P, \mu) \stackrel{\alpha}{\rightarrow} H^{1}(F, \mu) \rightarrow H^{1}(R, \mu)^{P} \rightarrow H^{2}(P, \mu) \rightarrow H^{2}(F, \mu) .
$$

The last term is zero because cd $F=1$. $\alpha$ is an isomorphism because $F$ was chosen to be minimal. Also, notice that $H^{1}(R, \mu)^{p}$ $=\operatorname{Hom}(R /[R, F], \mu)$. Thus

$$
\operatorname{Hom}(R /[R, F], \mu) \approx H^{2}(P, \mu) .
$$

If $P$ is not free, $R \neq(1)$, and Lemma 5 shows that $R /[R, F]$ is not torsion. Pontryagin duality now shows that $H^{2}(P, \mu)$ contains a nontrivial divisible subgroup (dual to the maximal torsion-free quotient of $R /[R, F] !)$.

Corollary 1. Let $K$ be a field whose Galois group $G_{K}$ is abelian. The $B(K)_{p}$ is divisible unless $p=2$ and $\sqrt{ }(-1) \notin K$. In the latter case $2 \mathrm{~B}(\mathrm{~K})_{2}$ is divisible.

Proof. Lemma 3 implies we may assume $G_{K}$ is a pro-p-group. Assume that all the $p^{n}$ th roots of unity are in $K$. Then, as in the proof of the theorem, $G_{K} \approx F / F_{1}$ for some free pro-p-group $F$. Hence $B(K)_{p}$ is dual to $F_{1} /\left[F_{1}, F\right]=F_{1} / F_{2}$ which is torsion free. It follows that $B(K)_{p}$ is divisible.

By Lemma 4 the corollary is proven unless $p=2$ and $\sqrt{ }(-1) \notin K$.

Let $L=K(\sqrt{ }(-1))$. Then $B(L)_{2}$ is divisible. Since $G_{L}$ is of index 2 in $G_{K}$, we have the maps res: $B(K) \rightarrow B(L)$ and cor: $B(L) \rightarrow B(K)$ arising from cohomology. The composition cor $\circ$ res $=$ multiplication by 2 . Thus

$$
B(K)_{2} \supset \operatorname{cor} B(L)_{2} \supset \text { cor o res } B(K)_{2}=2 B(K)_{2} \text {. }
$$

From the fact that a homomorphic image of a divisible group is divisible, it follows readily that $2 \mathrm{~B}(K)_{2}$ is divisible.

Corollary 2. Assume all the $p^{n}$ th roots of unity are in the ground field $K$, and that $G_{K}$ is pro-nilpotent. Then either $B(K)_{p}=(0)$ or $B(K)_{p}$ contains a nontrivial divisible subgroup.

Proof. Let $P$ be a $p$-Sylow subgroup of $G_{K} . B(K)_{p}=H^{2}\left(G_{K}, \mu\right)$, and by Lemma $3 H^{2}\left(G_{K}, \mu\right)$ is either $(0)$ or $H^{2}(P, \mu)$. Suppose the latter case holds. Since $P$ is a direct factor of $G_{K}$, it is isomorphic to the Galois group of the maximal p-extension of $K$. Thus either $H^{2}(P, \mu)=(0)$ or it contains a nontrivial divisible subgroup. 


\section{BIBLIOGRAPHY}

1. Maurice Auslander and Armand Brumer, On the Brauer group of commutative rings, Proc. Roy. Soc. Netherlands (to appear).

2. John Labute, Demuskin groups of rank $\aleph_{0}$, Bull. Soc. Math. France 94 (1966), 211-244.

3. J.-P. Serre, Cohomologie galoisienne, Springer-Verlag, Berlin, 1964.

Columbia University and

BROWN UNIVERSITY 